\title{
Bioelectrocatalysis on Anodized Epitaxial Graphene and Conventional Graphitic Interfaces
}

Mikhail Vagin, Alina Sekretareva, Anna Håkansson, Tihomir Iakimov, Ivan Gueorguiev Ivanov, Mikael Syväjärvi, Rositsa Yakimova, Ingemar Lundström and Mats Eriksson

The self-archived postprint version of this journal article is available at Linköping University Institutional Repository (DiVA):

http://urn.kb.se/resolve?urn=urn:nbn:se:liu:diva-159728

N.B.: When citing this work, cite the original publication.

Vagin, M., Sekretareva, A., Håkansson, A., Iakimov, T., Ivanov, I. G., Syväjärvi, M., Yakimova, R., Lundström, I., Eriksson, M., (2019), Bioelectrocatalysis on Anodized Epitaxial Graphene and Conventional Graphitic Interfaces, CHEMELECTROCHEM, 6(14), 3791-3796.

https://doi.org/10.1002/celc.201900587

Original publication available at:

https://doi.org/10.1002/celc.201900587

Copyright: Wiley

http://eu.wiley.com/WileyCDA/ 


\title{
Bioelectrocatalysis on anodized epitaxial graphene and conventional graphitic interfaces
}

\author{
Mikhail Yu. Vagin ${ }^{1,2 * \#}$, Alina N. Sekretaryova ${ }^{1,3,4 \#}$, Anna Håkansson ${ }^{1,2}$, Tihomir Iakimov ${ }^{1,5}$, \\ Ivan G. Ivanov $^{1}$, Mikael Syväjärvi ${ }^{1,5}$, Rositsa Yakimova ${ }^{1,5}$, Ingemar Lundström ${ }^{1}$, Mats Eriksson ${ }^{1}$ \\ ${ }^{1}$ Department of Physics, Chemistry and Biology, Linköping University, 58183, Linköping, \\ Sweden; e-mail: mikhail.vagin@liu.se \\ ${ }^{2}$ Laboratory of Organic Electronics, Department of Science and Technology, Linköping \\ University, 60174, Norrköping, Sweden \\ ${ }^{3}$ Department of Chemistry, Stanford University, Stanford, CA 94305-5080, USA \\ ${ }^{4}$ Department of Chemistry 'Ångström', Uppsala University, Lägerhyddsvägen 1, 75120 Uppsala, \\ Sweden; e-mail: alina.sekretareva@kemi.uu.se \\ ${ }^{5}$ Graphensic AB, Teknikringen 1F, SE-58330 Linköping, Sweden \\ \# These authors contributed equally
}

\begin{abstract}
Graphitic materials exhibit significant anisotropy due to the difference in conductivity in a single layer and between adjacent layers. This anisotropy is manifested on epitaxial graphene (EG) which can be manipulated on the nanoscale in order to provide tailor-made properties. Insertion of defects into the EG lattice was utilized here for controllable surface modification with a model biocatalyst and the properties were quantified by both electrochemical and optical methods. A comparative evaluation of the electrode reaction kinetics on the enzyme-modified 2-D material vs conventional carbon electrode materials revealed a significant enhancement of mediated bioelectrocatalysis at the nanoscale.
\end{abstract}

Keywords: epitaxial graphene, laccase bioelectrocatalysis, voltammetry, graphitic materials

*Corresponding authors 


\section{INTRODUCTION}

Graphene is a 2D nanomaterial offering the possibility to explore electrochemical processes taking place on graphitic materials at the simplest level of structural organization. A significant difference of the density of electronic states at in-plane and out-of-plane conduction in graphene gives rise to two distinct contributions (basal and edge plane respectively) to the performance of all graphitic materials yielding an anisotropy $[1,2]$. The use of flat graphene sheets with controllable exposure of the basal plane obtained by chemical vapor deposition [3-5] or by epitaxial annealing of crystalline structures $[6,7]$ allows decoupling of these two contributions to the conductivity of graphite.

Enzyme immobilization onto graphene monolayers has been realized for various applications, such as biosensing [8], enzyme activity monitoring [9, 10] and supramolecular enzyme complexes assembly $[11,12]$. However, the number of such studies is limited in comparison with the vast literature available on the use of bulk materials based on graphene flakes for biocatalyst immobilization [13]. The inertness of a graphene monolayer to reactions in the out-of-plane direction is the main reason for this discrepancy. Thus, there is an increasing interest in the development of approaches for graphene functionalization to optimize its role for biocatalysis [14]. Anodization is a technique to induce defects into graphite [15] which can be utilized for graphene monolayer treatment yielding an increase of both electrical charge storage capacity and faradaic activity [16]. The anodization as a result of oxygen evolution reaction [17] generates defects in the graphene lattice [16] resulting in fracturing $[18,19]$ and removes contaminants, hindering the kinetics of surface-sensitive redox processes, from the surface [20].

Here we explore the possibility to use anodization induced defects in the graphene lattice of EG to be used for covalent coupling of a model biocatalyst. The appearance of carboxylic groups available for further modification has been quantified by both electrochemical and optical methods. Supported by physical methods, the evaluation of the electrode reaction kinetics on laccase-modified graphitic materials revealed a significant enhancement in mediated 
bioelectrocatalysis efficiency on the EG monolayer in comparison with conventional graphitic electrode materials such as screen-printed graphite and glassy carbon.

\section{EXPERIMENTAL}

\section{Reagents}

All inorganic salts, catechol, toluidine blue (TB), N-hydroxysulfosuccinimide (NHS) sodium salt, 1-(3-dimethylaminopropyl)-3 ethylcarbodiimide (EDC), 2,2'-Azino-bis(3-ethylbenzothiazoline6-sulfonic acid) (ABTS), laccase from Trametes versicolor $\left(\geq 10 \mathrm{U} \mathrm{mg}^{-1}\right)$ were purchased from Sigma (Sweden) and used as received. Experiments were carried out with Milli-Q water from a Millipore Milli-Q system.

\section{Samples and processes}

An Autolab type III potentiostat (Autolab, EcoChemie, Netherlands) was used for the electrochemical measurements. $\mathrm{An} \mathrm{Ag} / \mathrm{AgCl}$ electrode in $3 \mathrm{M} \mathrm{KCl}$ and a platinum wire were used as reference and counter electrodes, respectively, for all measurements.

Screen-printed electrode (SPE) systems with a graphite working electrode (diameter $4.0 \mathrm{~mm}$ ) were purchased from Dropsens (Spain). Glassy carbon electrodes (GCE) (diameter $3.0 \mathrm{~mm}$ ) were purchased from CHI Instruments (USA).

The samples of nominally monolayer EG on $\mathrm{SiC}$ (substrate area $7 \times 7 \mathrm{~mm}^{2}$ and thickness $0.4 \mathrm{~mm}$ ) and the electrochemical cell employed for the measurements wereobtained from Graphensic AB.Graphene was grown on the Si-face of the $\mathrm{SiC}$ substrates [21]. Optical reflectance measurements (Figure S3) show that the two different samples we investigated had 80 and 55\% of monolayer coverage, respectively, while the rest is small patches of bilayer graphene. The cell consisted of a cup of $300 \mu \mathrm{l}$ with a $2 \mathrm{~mm}$ diameter hole with a Vitron o-ring on the bottom. The EG sample was fixed under the hole with the o-ring using screws on the lid. A dry contact to the 
EG was formed by an aluminum adhesive. The mounted EG sample was kept inside the cell during all wet measurements and procedures in order to avoid sample drift.

\section{Pretreatment of electrodes}

Pretreatments of the electrodes were done according to protocols optimized for the different electrode materials $[16,19,22]$. The GCEs were successively polished with alumina powders (1.0; 0.3 and $0.05 \mu \mathrm{m}$ ) followed by ultrasonication in distilled water. The anodization was carried out in $0.05 \mathrm{M}$ phosphate buffer solution ( $\mathrm{pH} 7.0$ ) with a $300 \mathrm{~s}$ min oxidation pulse at $1.8 \mathrm{~V}$ followed by a $60 \mathrm{~s}$ reduction pulse at $-1.5 \mathrm{~V}[22]$.

The SPEs were activated by anodization of the bare electrode at $1.4 \mathrm{~V}$ for $90 \mathrm{~s}$ in saturated $\mathrm{Na}_{2} \mathrm{CO}_{3}$. The mounted EG electrode anodization was carried out in a $0.1 \mathrm{M} \mathrm{KNO}_{3}$ solution for $300 \mathrm{~s}$ in a continuous pulsed mode (a $0.5 \mathrm{~s}$ pulse at $2 \mathrm{~V}$ followed by a $0.125 \mathrm{~s}$ pulse at $0.1 \mathrm{~V}$ ) [16].

\section{Surface analysis by toluidine blue quantification}

The TB method $[23,24]$ was utilized for estimation of the density of carboxylic groups on the electrode surfaces. Briefly, the pretreated electrodes were soaked in $0.5 \mathrm{mM}$ TB in $0.1 \mathrm{mM} \mathrm{NaOH}$ overnight for TB binding. Then the electrode surface was washed with $0.1 \mathrm{mM} \mathrm{NaOH}$ solution and pure water twice. The amount of TB molecules attached to the electrode surface was estimated by optical and electrochemical measurements assuming 1:1 stoichiometry of the TB-carboxylic group complex. In the optical protocol, $100 \mu \mathrm{L}$ of $50 \%$ acetic acid was added to remove surfacebound TB. The optical density of the solution was measured at $630 \mathrm{~nm}$ using a microplate reader. The concentration of released TB was determined using a calibration plot. For the electrochemical protocol, a scan rate study of the voltammetric response of the TB-modified electrodes allowed an estimation of the surface coverage [25]. 


\section{Surface analysis by physical methods}

The monolayer and bilayer regions of EG were distinguished by optical reflectance measurements [26] where a lens with 100X magnification and a solid-state laser at $532 \mathrm{~nm}$ were employed. The scattered light was analyzed using a monochromator (Jobin-Yvon, model HR460) equipped with a CCD camera. The laser power at the sample was $\sim 17 \mathrm{~mW}$ focused to a spot with a diameter of approximately $0.85 \mu \mathrm{m}$.

Atomic force microscopy (AFM) in tapping mode was carried out with a Dimension 3100SPM (Veeco Instruments) equipped with acoustic/vibration isolation system and the VT-102 vibration isolation table using Micromasch NSC14 cantilevers. Nanoscope Analysis software was utilized for AFM data analysis.

\section{Laccase immobilization}

The coupling between laccase and the functional groups of the pretreated electrodes was done via EDC/NHS chemistry [27]. A mixture of $5 \mathrm{mM}$ NHS and $5 \mathrm{mM}$ EDC solutions in water was dropped on the surface of the pretreated electrodes and left for 2 hours at room temperature for the reaction to occur. After rinsing with distillated water, $100 \mathrm{U} \mathrm{mL}^{-1}$ laccase solution in water was dropped on the surface of the electrodes and left at $4{ }^{\circ} \mathrm{C}$ overnight. The non-immobilized enzyme was removed by repeated washing with deionized water. The laccase-immobilized modified electrodes were stored at $4^{\circ} \mathrm{C}$ in $0.1 \mathrm{M}$ acetate buffer solution at $\mathrm{pH} 5.0$ when not used.

\section{Estimation of the laccase surface concentration}

To measure the laccase enzyme concentration on the electrode surface, spectroscopic measurements of the immobilized laccase activity were performed with 2,2'-azino-bis(3ethylbenzothiazoline-6-sulphonic acid) (ABTS) as a substrate using a standard protocol [28]. 
Briefly, $100 \mu \mathrm{l}$ of $0.8 \mathrm{mM}$ ABTS solution in $100 \mathrm{mM}$ acetate buffer at $\mathrm{pH} 5.0$ was applied to the electrode surface during a certain time and the solution absorbance at $420 \mathrm{~nm}\left(\varepsilon_{420}\right.$ ABTS $=36000$ $\mathrm{M}^{-1} \mathrm{~cm}^{-1}$ ) was measured. The reaction times were 5, 4, 5, 10, 7, 5 and 0 (background, no ABTS solution added) minutes for each electrode. Assuming that the activity of $1 \mathrm{mg}$ of laccase is 10.5 $\pm 0.4 \mathrm{U} \mathrm{mg}^{-1}$ [29], the concentration of the immobilized enzyme was calculated from the increase in solution absorbance.

\section{RESULTS AND DISCUSSION}

Anodization of different carbon electrodes by applying high oxidative potentials was performed in order to create oxygenated groups on the electrode surface for subsequent covalent attachment of a model biocatalyst. Laccase was used in the work presented here and is an enzyme containing copper in its active site, which catalyzes the oxidation of various phenolic compounds [30] concomitant with a four-electron reduction of molecular oxygen to water without the intermediate formation of hydrogen peroxide. The enzyme has a broad substrate specificity [31], and is therefore widely utilized in electroanalysis [32].

EG anodization was carried out in accordance with the procedure recently reported by our group, which led to the formation of defects in the graphene lattice [16]. SPEs and GCEs were anodized using a published approach [22] where anodization times and applied potentials were optimized based on the appearance of redox peaks in differential pulse voltammograms (DPV). The differential pulse voltammetry displayed distinct redox peaks that appeared on all electrode materials after anodization (Fig. 1). Such redox activity is assigned to oxygenated groups that appear on the surfaces during the pretreatment $[22,33,34]$. The surface redox process occurred at about $-0.1 \mathrm{~V}$ for the SPEs and the GCEs while the EG samples showed an anodic shift of approximately $+0.15 \mathrm{~V}$ of the formal potential compared to the other two electrodes. The anodization resulted in a growth of the background currents on all electrode materials due to a reactivity enhancement towards faradaic processes and an increase of the capacitive contributions 
due to the increased surface roughness induced by the process. The voltammetric response of EG showed the smallest currents illustrating the ultimate smoothness of this electrode structure, which is beneficial for electroanalysis [35].

Coupling of the created oxygenated groups with TB was utilized to estimate the concentration of these groups using both electrochemical and optical $[23,24]$ quantification methods. Voltammetry on anodized TB-modified electrodes revealed a quasi-reversible redox process (Fig. S1) of the immobilized dye molecules. Assuming that the observed signals are due to a surface confined redox process, the linear regions of the scan rate vs redox peak current plots (Insets in Fig. S1) can be used for an estimation of the TB surface coverage $\left(\Gamma_{\text {TOTAL }}\right)[25]$ :

$\Gamma_{T O T A L}=\frac{4 R T I_{P}}{v A F n^{2}}$

where $I_{P}$ is the cathodic peak current, $v$ is the scan rate, $A$ is the electrode surface area and all other symbols have their usual meanings.

The optical read-out using a standard TB method protocol [23, 24] allowed an independent quantification of TB molecules bound to carboxylic groups. As can be seen from Table 1, the surface coverage of the GCEs and the SPEs assayed by spectroscopy and electrochemistry are in good agreement. The TB method was not applied to the EG due to a limited number of samples and the inability to regenerate the anodized surface after binding of the TB molecules. Instead, the amount of oxygenated groups on EG samples was estimated from DPVs. Considering only the Faradaic part (the peak current measured with respect to the extrapolation of the background current flowing on either side of the peak in the voltammogram) of the DPV peak currents corresponding to oxygenated groups, the surface coverage of oxygenated groups can be estimated using the following equation [36]:

$i_{p f}=\frac{\text { const } * \Gamma}{2-B \Gamma}$

where $\Gamma$ is the surface coverage of redox active groups, $i_{p f}$ is the peak current due to the Faradaic contribution, const $=\frac{\Delta E n^{2} F^{2}}{\tau e \times 2 R T}, \Delta E$ is the magnitude of the potential pulse, $\tau$ is the time after the 
pulse application when the current is measured, $e=2.718, B$ is a curve fitting parameter that can be determined from cyclic voltammetry, and all other symbols have their usual meanings. Fitting of the experimental data for different electrode surfaces to eq. (2) (Fig. S2) gives a coverage of the anodized EG of $\sim 1 \mathrm{pmol} \mathrm{cm}^{-2}$. The DPV peak heights obtained for EG samples are comparable to those of polished GCEs, giving an estimation of the oxygenated group density of 2-5 pmol cm $\mathrm{cm}^{-2}$. Thus, the density of oxygenated groups on the EG samples is estimated to be in the range of 1-5 pmol cm $\mathrm{cm}^{-2}$.

Covalent coupling via EDC/NHS chemistry with the carboxylic groups of the anodized electrodes was utilized for laccase immobilization [27]. Surface analysis of the laccase-modified EG by AFM (Fig. 2) showed the presence of three morphological contributions with different sizes: the uniformly-distributed small $(0.5-3 \mathrm{~nm})$ and moderate scale features $(7-13 \mathrm{~nm})$ as well as individual objects of large scale $(40-45 \mathrm{~nm})$. The observed surface morphology differs significantly from the smooth character of the pristine EG which is characterized by uniformly-distributed features on a scale smaller than $0.2 \mathrm{~nm}$.

The presence of the enzyme on the electrode surface results in direct electron transfer (DET) observed as an increase of cathodic currents after addition of oxygen to the background electrolyte [37]. In the presence of a shuttling substrate (e.g. catechol), which can undergo both an enzymatic oxidation and re-reduction on the electrode surface, mediated electron transfer (MET) takes place [38] as substrate-mediated oxygen reduction yielding further increase of the cathodic currents. Both DET and MET were observed on the laccase-modified anodized electrode structures by independent voltammetry measurements in the absence and presence of oxygen and catechol. SPE had the highest currents of DET, recorded by background-corrected voltammetry in catechol-free oxygen-containing buffer. Interestingly, EG showed the smallest DET current densities, which are comparable with the characteristics of laccase-associated electrocatalysis on reduced graphene oxide [39]. The smallest magnitude of DET currents might be rationalized as a general roughness 
effect [40]. Due to the high roughness and the contribution from graphite particles in the printed ink [41], SPE showed largest DET efficiency.

The addition of catechol as an electron-shuttling substrate (Fig. 3) led to a current increase due to the MET on all laccase-modified electrode structures. The rate constants of the mediated electrode reactions were calculated according to [42]:

$\frac{1}{i_{c a t}}=\frac{1}{2 F k^{0} \Gamma_{E}^{0}} \frac{1}{[Q]_{0}}+$ const

where $i_{c a t}$ - the steady-state reduction current, $F$ - Faraday constant, $k^{0}$ - mediated electron transfer rate constant, $\Gamma_{E}^{0}$ - concentration of enzyme on the electrode surface, $[Q]_{0}$ - concentration of mediator in solution. The concentration of the active enzyme on the electrode surface was estimated from spectroscopic measurements of the enzyme activity using ABTS as a substrate. $\Gamma^{0}{ }_{E}$ estimated from Fig S3 indicates that the number of bound active enzyme molecules to anodized EG was considerably lower compared to the other two electrodes, in accordance with the lower amount of anodized groups on EG as indicated by DPV. Using the obtained values of $\Gamma^{0}$, the values of the mediated electron transfer rate constants were estimated from the slopes of the $1 / i_{\text {cat }}$ vs $1 /[Q]_{0}$ plots. The result is that the rate constant is considerably larger for anodized EG than for GCE and SPE (Fig. 4). This is coherent with the previously observed dramatic acceleration of the catechol redox process due to anodization of glassy carbon yielding a significant increase of the heterogeneous rate constant[33]. An increase of the bilayer content of the EG (Fig. S4) led to a decrease of the amount of immobilized enzyme and a corresponding change of the MET rate constant confirming the inactivity of multilayered regions of EG towards anodization [16]. Importantly, among the evaluated electrode materials EG showed a higher efficiency of conversion $\left(k^{0}\right)$ of the enzymatic process into a recorded current via the mediator redox process. This illustrates the diverse activity of modified carbon surfaces towards faradaic reactions $[16,18,19]$. The high smoothness of EG, illustrated by the smallest values of capacitive currents in comparison with the 
other graphitic interfaces, appears to be very favorable for maximization of faradaic-to-capacitive current (signal-to-noise) ratio and for accessibility towards surface modification by defects reactive in the redox process of shuttling mediators. In other words, the surface-normalized electrode activity towards faradaic reactions established at the nanoscale is a key element in the redox transducer performance.

\section{CONCLUSIONS}

Biocatalyst immobilization on EG was quantified for the first time. The presence of the enzyme molecules immobilized on the anodized EG was observed by AFM, optical and electrochemical methods. In contrast to the minor direct communication between the biocatalyst and the interface in the case of EG, the mediated bioelectrocatalysis revealed a higher efficiency on the 2-D material compared to the other carbon interfaces, illustrating the interplay between surface roughness and anodization-gained reactivity towards redox reactions. Anodized EG is an effective substrate for electrocatalysis since a low density of bound active enzyme molecules shows a high activity per molecule. 


\section{REFERENCES}

[1] D.A.C. Brownson, D.K. Kampouris, C.E. Banks, Chem. Soc. Rev. 2012, 41, 6944-6976.

[2] R.L. McCreery, Chem. Rev. 2008, 108, 2646-2687.

[3] A. Ambrosi, A. Bonanni, Z. Sofer, M. Pumera, Nanoscale 2013, 5, 2379-2387.

[4] X. Huang, Z. Zeng, Z. Fan, J. Liu, H. Zhang, Adv. Mater. 2012, 24, 5979-6004.

[5] A. Reina, X.T. Jia, J. Ho, D. Nezich, H.B. Son, V. Bulovic, M.S. Dresselhaus, J. Kong, Nano Lett. 2009, 9, 30-35.

[6] K.V. Emtsev, A. Bostwick, K. Horn, J. Jobst, G.L. Kellogg, L. Ley, J.L. McChesney, T. Ohta, S.A. Reshanov, J. Roehrl, E. Rotenberg, A.K. Schmid, D. Waldmann, H.B. Weber, T. Seyller, Nat. Mater. 2009, 8, 203-207.

[7] R. Yakimova, C. Virojanadara, D. Gogova, M. Syvajarvi, D. Siche, K. Larsson, L.I. Johansson, Analysis of the Formation Conditions for Large Area Epitaxial Graphene on SiC Substrates, in: A.J. Bauer, P. Friedrichs, M. Krieger, G. Pensl, R. Rupp, T. Seyller (Eds.) Silicon Carbide and Related Materials 2009, Pts 1 and 22010, pp. 565-568.

[8] M.B. Lerner, F. Matsunaga, G.H. Han, S.J. Hong, J. Xi, A. Crook, J.M. Perez-Aguilar, Y.W. Park, J.G. Saven, R.Y. Liu, A.T.C. Johnson, Nano Lett. 2014, 14, 2709-2714.

[9] H.K. Lee, J. Heo, S. Myung, I.S. Shin, T.H. Kim, Electroanal. 2017, 29, 1749-1754.

[10] K. Islam, R. Chand, D. Han, I.S. Shin, Y.S. Kim, J. Electrochem. Soc. 2014, 161, B261-B264.

[11] W.R. Yang, Q. Ren, Y.N. Wu, V.K. Morris, A.A. Rey, F. Braet, A.H. Kwan, M. Sunde, Biopolymers, 2013, 99, 84-94.

[12] A. Alshammari, M.G. Posner, A. Upadhyay, F. Marken, S. Bagby, A. Ilie, ACS Appl. Mater. \& Inter. 2016, 8, 21077-21088.

[13] Y.Y. Shao, J. Wang, H. Wu, J. Liu, I.A. Aksay, Y.H. Lin, Electroanal. 2010, 22, 1027-1036. 
[14] V. Georgakilas, M. Otyepka, A.B. Bourlinos, V. Chandra, N. Kim, K.C. Kemp, P. Hobza, R. Zboril, K.S. Kim, Chem. Rev. 2012, 112, 6156-6214.

[15] C.Y. Lee, A.M. Bond, Anal. Chem. 2009, 81, 584-594.

[16] M.Y. Vagin, A.N. Sekretaryova, I.G. Ivanov, A. Håkansson, T. Iakimov, M. Syväjärvi, R. Yakimova, I. Lundström, M. Eriksson, Electrochim. Acta 2017, 238, 91-98.

[17] M. Cattelan, M. Vagin, N.A. Fox, I. Ivanov, I. Shtepliuk, R. Yakimova, Nanotechnology, accepted (2019).

[18] E. Dubuisson, Z. Yang, K.P. Loh, Anal. Chem. 2011, 83, 2452-2460.

[19] C.X. Lim, H.Y. Hoh, P.K. Ang, K.P. Loh, Anal. Chem. 2010, 82, 7387-7393.

[20] A.N. Patel, M.G. Collignon, M.A. O'Connell, W.O.Y. Hung, K. McKelvey, J.V. Macpherson, P.R. Unwin, JACS 2012, 134, 20117-20130.

[21] T. Burnett, R. Yakimova, O. Kazakova, Mapping of Local Electrical Properties in Epitaxial Graphene Using Electrostatic Force Microscopy, Nano Lett. 2011, 11, 2324-2328.

[22] T. Nagaoka, T. Yoshino, Surface-properties of electrochemically pretreated glassy-carbon, Anal. Chem. 1986, 58, 1037-1042.

[23] M. Ardhaoui, M.H. Zheng, J. Pulpytel, D. Dowling, C. Jolivalt, F.A. Khonsari, Bioelectrochem. 2013, 91, 52-61.

[24] C. Chollet, C. Chanseau, B. Brouillaud, M.C. Durrieu, Biomol. Eng. 2007, 24, 477-482.

[25] R.G. Compton, C.E. Banks, Understanding Voltammetry, World Scientific2007.

[26] I.G. Ivanov, J.U. Hassan, T. Iakimov, A.A. Zakharov, R. Yakimova, E. Janzen, Carbon 2014, $77,492-500$.

[27] D. Quan, W.S. Shin, Mat. Sci. Eng. C, 2004, 24, 113-115.

[28] C. Johannes, A. Majcherczyk, J. Biotechnol. 2000, 78, 193-199.

[29] A.N. Sekretaryova, A.V. Volkov, I.V. Zozoulenko, A.P.F. Turner, M.Y. Vagin, M. Eriksson, Anal. Chim. Acta 2016, 907, 45-53.

[30] H.P. Call, I. Mucke, J. Biotechnol. 1997, 53, 163-202. 
[31] R. Solna, P. Skladal, Electroanal. 2005, 17, 2137-2146.

[32] M.L. Mena, V. Carralero, A. Gonzalez-Cortes, P. Yanez-Sedeno, J.M. Pingarron, Electroanal. 2005, 17, 2147-2155.

[33] G.E. Cabaniss, A.A. Diamantis, W.R. Murphy, R.W. Linton, T.J. Meyer, JACS 1985, 107, $1845-1853$

[34] J. Wang, M. Pedrero, H. Sakslund, O. Hammerich, J. Pingarron, Analyst 1996, 121, 345-350.

[35] C.X. Lim, H.Y. Hoh, P.K. Ang, K.P. Loh, Anal. Chem. 2010, 82, 7387-7393.

[36] A.P. Brown, F.C. Anson, Anal. Chem. 1977, 49, 1589-1595.

[37] L. Gorton, A. Lindgren, T. Larsson, F.D. Munteanu, T. Ruzgas, I. Gazaryan, Anal. Chim. Acta 1999, 400, 91-108.

[38] B. Haghighi, L. Gorton, T. Ruzgas, L.J. Jonsson, Anal. Chim. Acta 2003, 487, 3-14.

[39] C. Di Bari, A. Goni-Urtiaga, M. Pita, S. Shleev, M.D. Toscano, R. Sainz, A.L. De Lacey, Electrochim. Acta 2016, 191, 500-509.

[40] C. Gutierrez-Sanchez, M. Pita, C. Vaz-Dominguez, S. Shleev, A.L. De Lacey, JACS 2012, $134,17212-17220$.

[41] J. Wang, M. Pedrero, H. Sakslund, O. Hammerich, J. Pingarron, Analyst 1996, 121, 345-350.

[42] C. Bourdillon, C. Demaille, J. Gueris, J. Moiroux, J.M. Saveant, JACS 1993, 115, 1226412269. 
Table 1. Estimated density of carboxylic groups and DPV peak heights.

\begin{tabular}{|c|c|c|c|c|c|}
\hline \multirow{2}{*}{\multicolumn{2}{|c|}{ Electrode }} & \multicolumn{3}{|c|}{$\mathrm{TB}, \mathrm{pmol} \mathrm{cm}-2$} & \multirow{2}{*}{$\begin{array}{c}\text { DPV peak height, } \\
\mathrm{mA} \mathrm{cm}^{-2}\end{array}$} \\
\hline & & spectroscopy & electrochemistry & estimated from & \\
\hline \multirow{2}{*}{ GCE } & polished & 2.5 & 2 & & 0.002 \\
\hline & anodized* & 31 & 28 & & 2.67 \\
\hline \multirow{2}{*}{ SPE } & bare & 6.6 & 8.0 & & 0.02 \\
\hline & anodized** & 18.8 & 24.5 & & 1.21 \\
\hline \multirow{2}{*}{ EG } & pristine & - & - & & 0.00003 \\
\hline & anodized $* * *$ & - & - & $1-5$ & 0.005 \\
\hline
\end{tabular}

$* 1.8 \mathrm{~V}$ for $300 \mathrm{~s},-1.5 \mathrm{~V}$ for $60 \mathrm{~s}(0.05 \mathrm{M} \mathrm{PBS}) ; * * 1.4 \mathrm{~V}$ for $90 \mathrm{~s}$ (saturated $\left.\mathrm{Na}_{2} \mathrm{CO}_{3}\right) ; * * 300$ seconds of pulsed pretreatment $\left(2 \mathrm{~V}\right.$ for $0.5 \mathrm{~s}, 0.1 \mathrm{~V}$ for $\left.0.125 \mathrm{~s} ; 0.1 \mathrm{M} \mathrm{KNO}_{3}\right)$.

Each value in the table represents an average of 3 measurements for GCEs and SPEs and 2 measurements for EG samples. 


\section{FIGURE LEGENDS}

Figure 1. Formation of oxygen functional groups on graphite surfaces. DPV curves were obtained on GCE (A), SPE (B) and EG (80\%monolayer) (C) before (dashed black lines) and after (solid red lines) anodization (pulse scan rate and amplitude: $10 \mathrm{mV} \mathrm{s}^{-1}$ and $50 \mathrm{mV}$, respectively; $0.05 \mathrm{M}$ phosphate buffer).

Figure 2. AFM images acquired on pristine (A) and laccase-modified, anodized (B) EG (55\% monolayer).

Figure 3. Direct and mediated laccase electrocatalysis on graphitic interfaces. Backgroundsubtracted linear sweep voltammetry on laccase-modified GCE (A), SPE (B) and EG (80\% monolayer) $(\mathbf{C})$ recorded before (dashed curves) and after additions of catechol (10, 50 and 100 $\mu \mathrm{M}, 0.1 \mathrm{M}$ acetate buffer, $1 \mathrm{mV} \mathrm{s}^{-1}$ scan rate).

Figure 4. A comparison of surface enzyme coverages and MET rate constants on the different carbon materials. Surface coverages of active enzyme molecules were estimated from the ABTS consumption data. Rate constants were obtained from Fig. 3 and Eq. (2). 80\% and 55\% refer to EG samples with $80 \%$ and $55 \%$ monolayer content, respectively. The rest is inactive bilayer EG. 


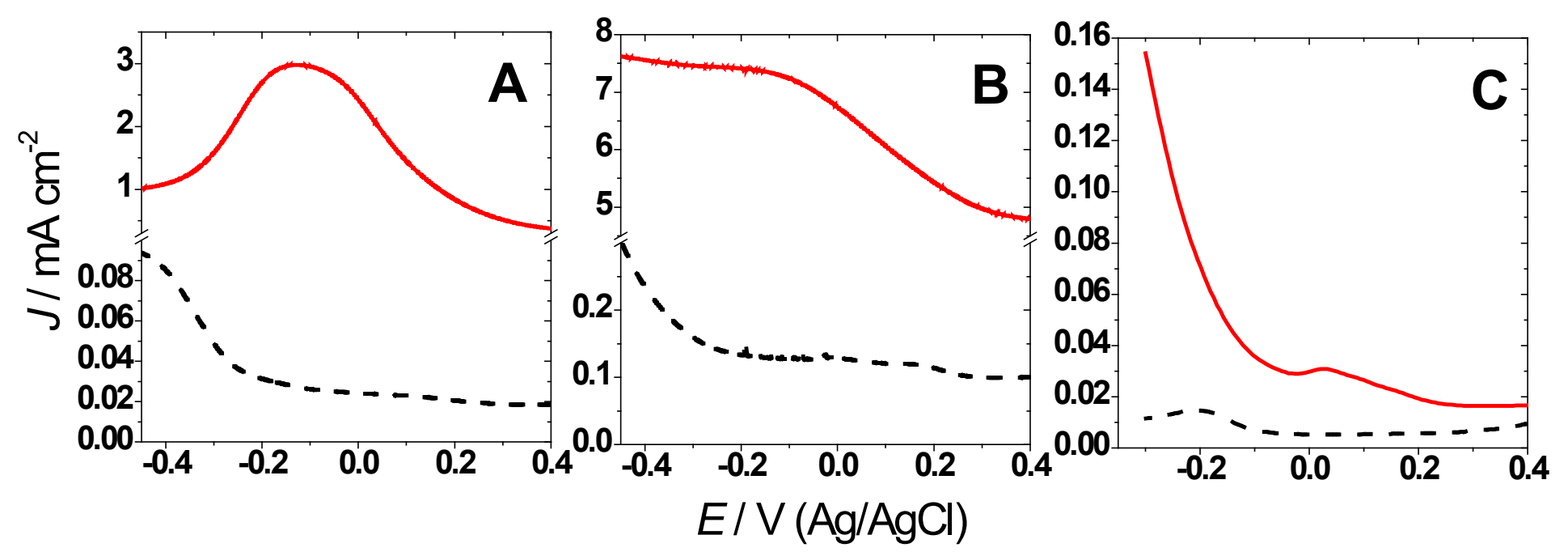

Figure 1. 


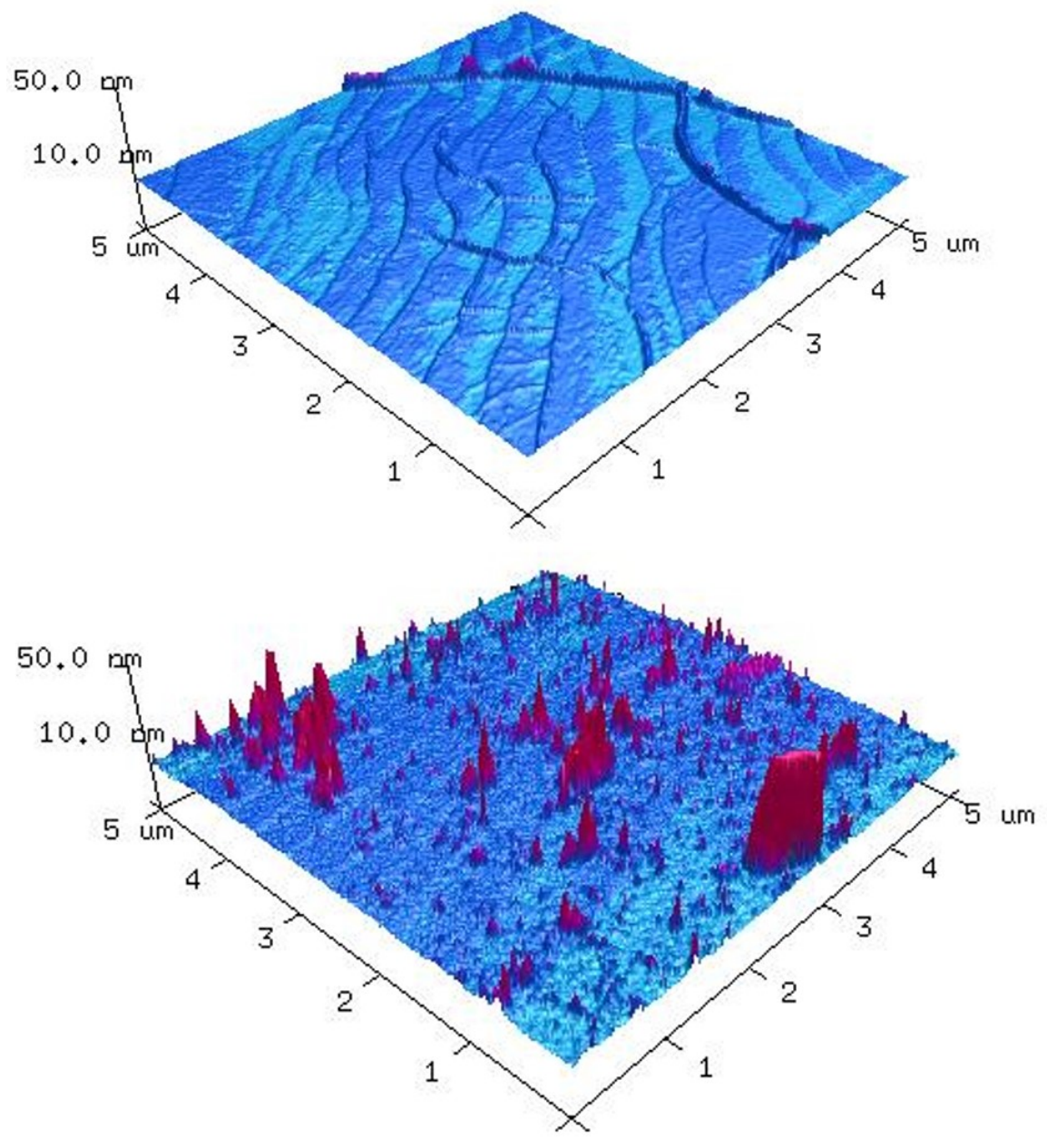

Figure 2. 


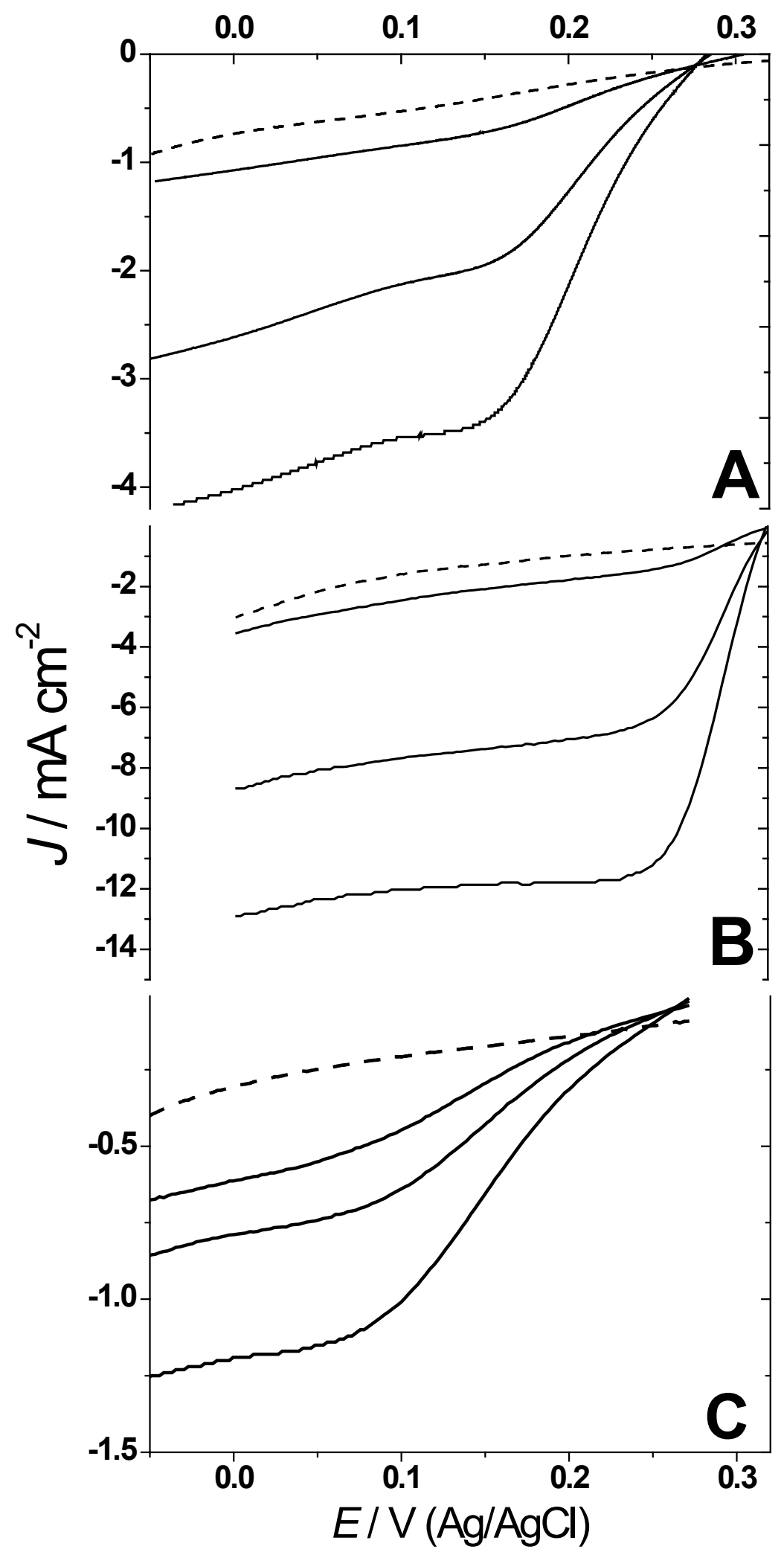

Figure 3. 


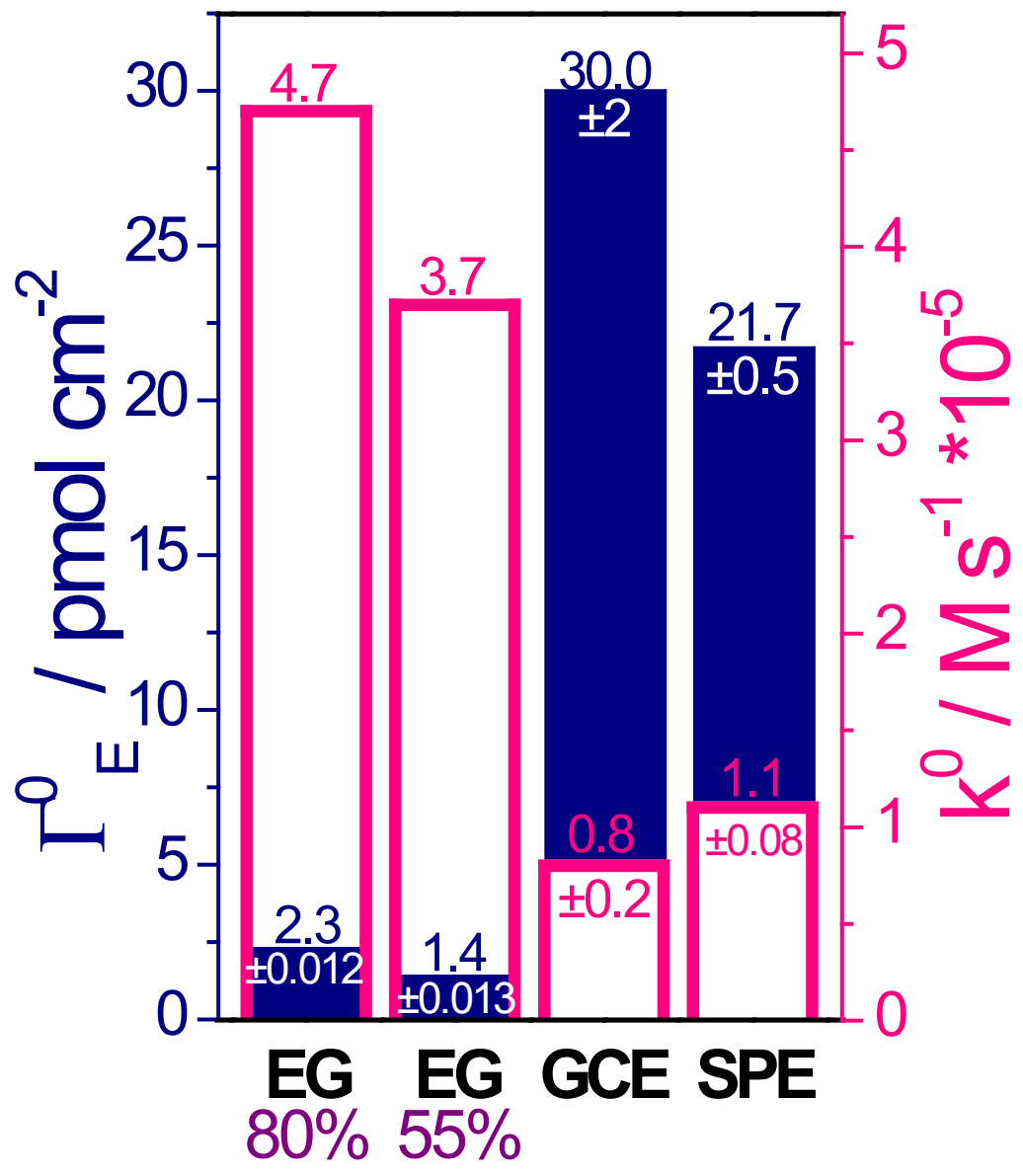

Figure 4. 


\section{Table of Contents}

Biocatalyst immobilization on EG was quantified for the first time.

The presence of the enzyme molecules immobilized on the anodized EG was observed by AFM, optical and electrochemical methods. The mediated bioelectrocatalysis revealed a higher efficiency on the 2-D material compared to the other carbon interfaces, illustrating the interplay between surface roughness and anodization-gained

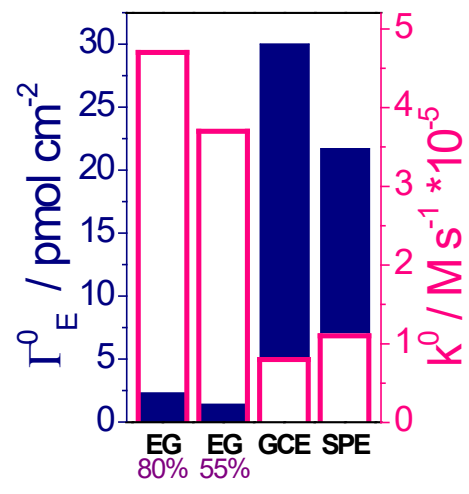
reactivity towards redox reactions. 


\title{
SUPPORTING INFORMATION
}

\section{Bioelectrocatalysis on anodized epitaxial graphene and conventional graphitic interfaces}

\begin{abstract}
Mikhail Yu. Vagin ${ }^{1,2 * \#}$, Alina N. Sekretaryova ${ }^{1,3,4 \#}$, Anna Håkansson ${ }^{1,2}$, Tihomir Iakimov ${ }^{1,5}$, Ivan G. Ivanov $^{1}$, Mikael Syväjärvi ${ }^{1,5}$, Rositsa Yakimova ${ }^{1,5}$, Ingemar Lundström ${ }^{1}$, Mats Eriksson ${ }^{1}$

${ }^{1}$ Department of Physics, Chemistry and Biology, Linköping University, 58183, Linköping,
\end{abstract} Sweden; e-mail: mikhail.vagin@liu.se

${ }^{2}$ Laboratory of Organic Electronics, Department of Science and Technology, Linköping University, 60174, Norrköping, Sweden

${ }^{3}$ Department of Chemistry, Stanford University, Stanford, CA 94305-5080, USA

${ }^{4}$ Department of Chemistry 'Ångström', Uppsala University, Lägerhyddsvägen 1, 75120 Uppsala, Sweden; e-mail: alina.sekretareva@kemi.uu.se

${ }^{5}$ Graphensic AB, Teknikringen 1F, SE-58330 Linköping, Sweden

\# These authors contributed equally 


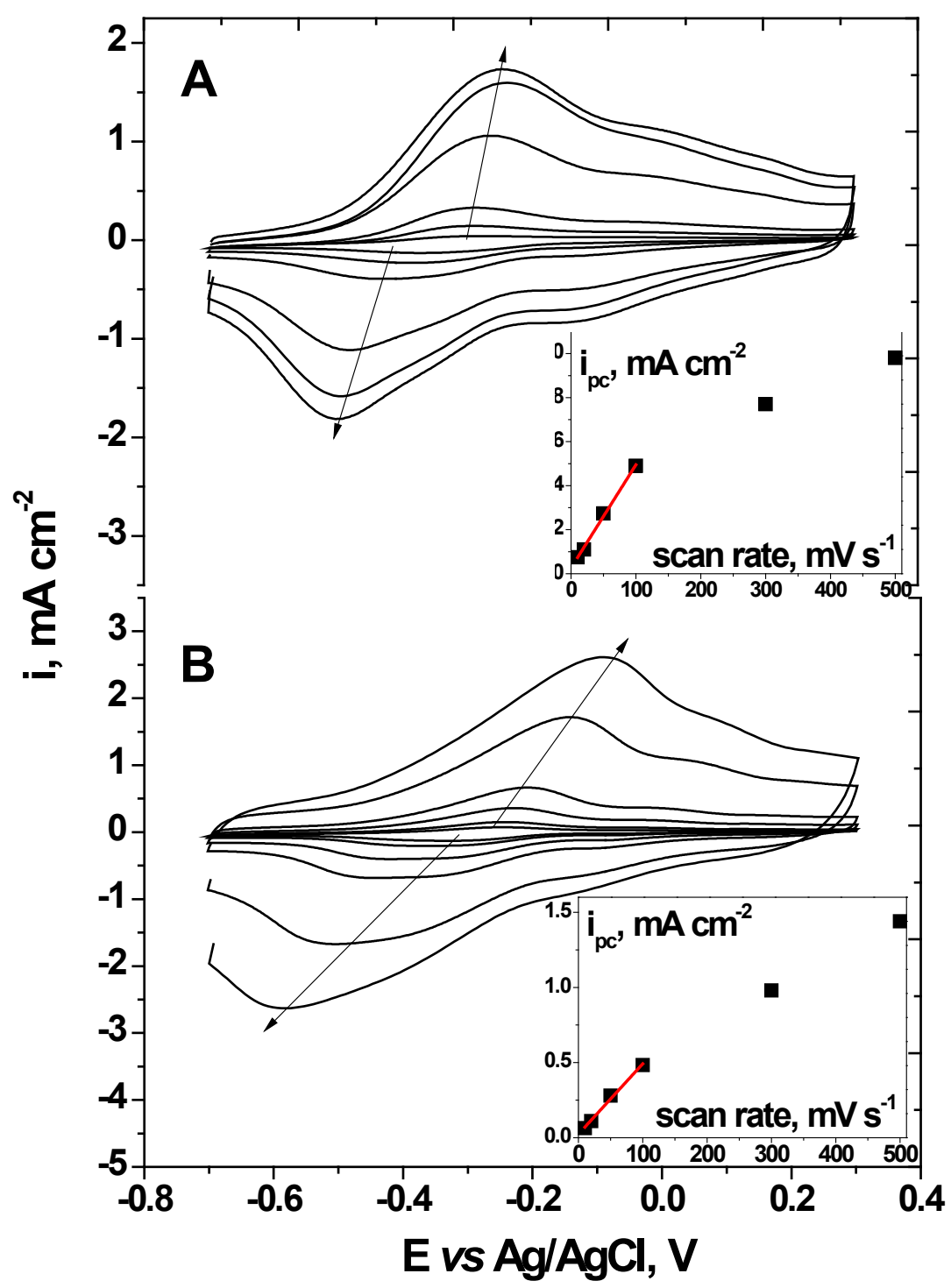

Figure S1. Voltammetry on TB-modified electrodes. Cyclic voltammograms of TB-modified GCE (A) and SPE (B) (0.05 M phosphate buffer, scan rates 10, 20, 50, 100, 300 and $\left.500 \mathrm{mV} \mathrm{s}^{-1}\right)$; Insets: Dependence of cathodic peak current on the scan rate. 


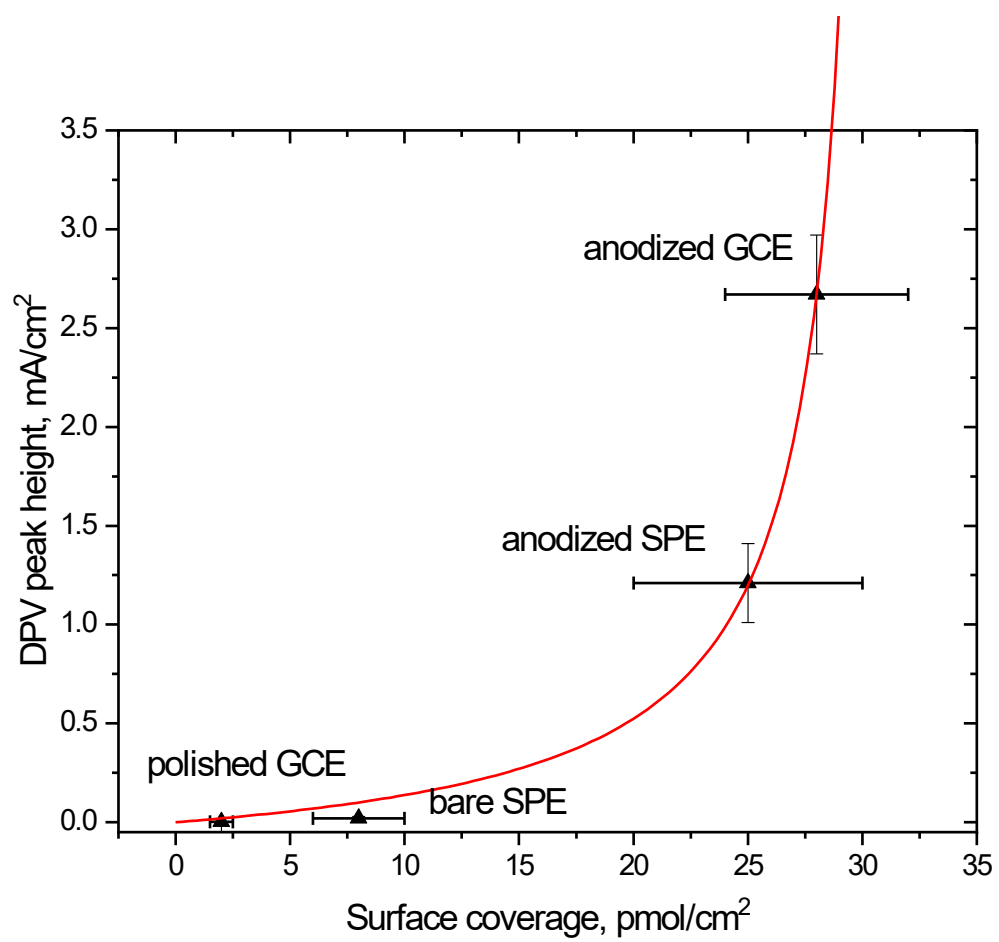

Figure S2. Dependence of the DPV peak heights on the surface coverage by oxygenated groups, experimental data (black dots represent mean values and, error bars show standard deviation based on 3 measurements) and data fit to eq. (2) (solid line). 


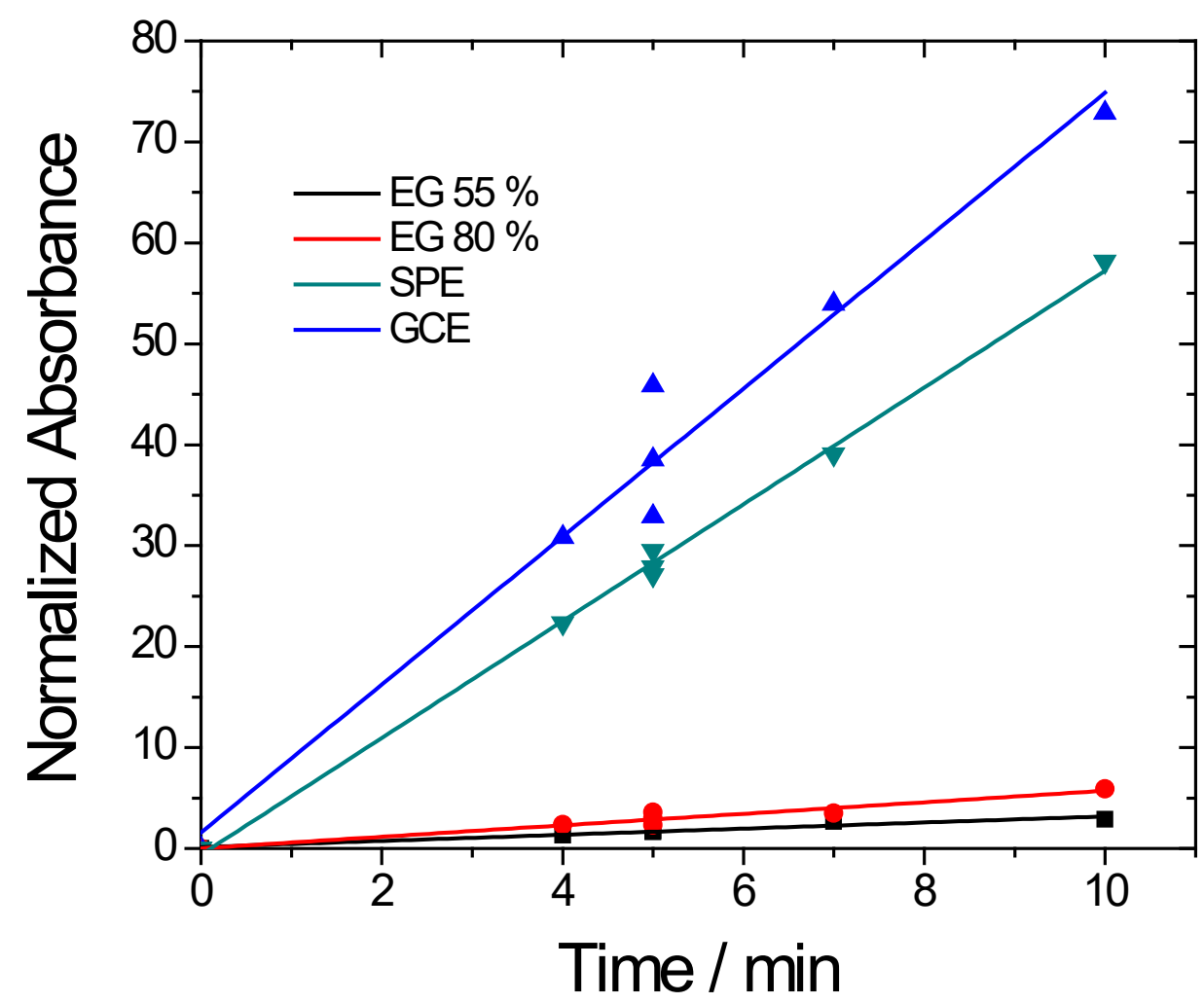

Figure S3. Absorbance of ABTS solutions at $420 \mathrm{~nm}$ collected after different reaction times. Normalized absorbance corresponds to the measured value of absorbance divided by the surface area of the electrode and dilution coefficient (10 for SPE and GCE, 1 for EG 80 and $55 \%$ ). 

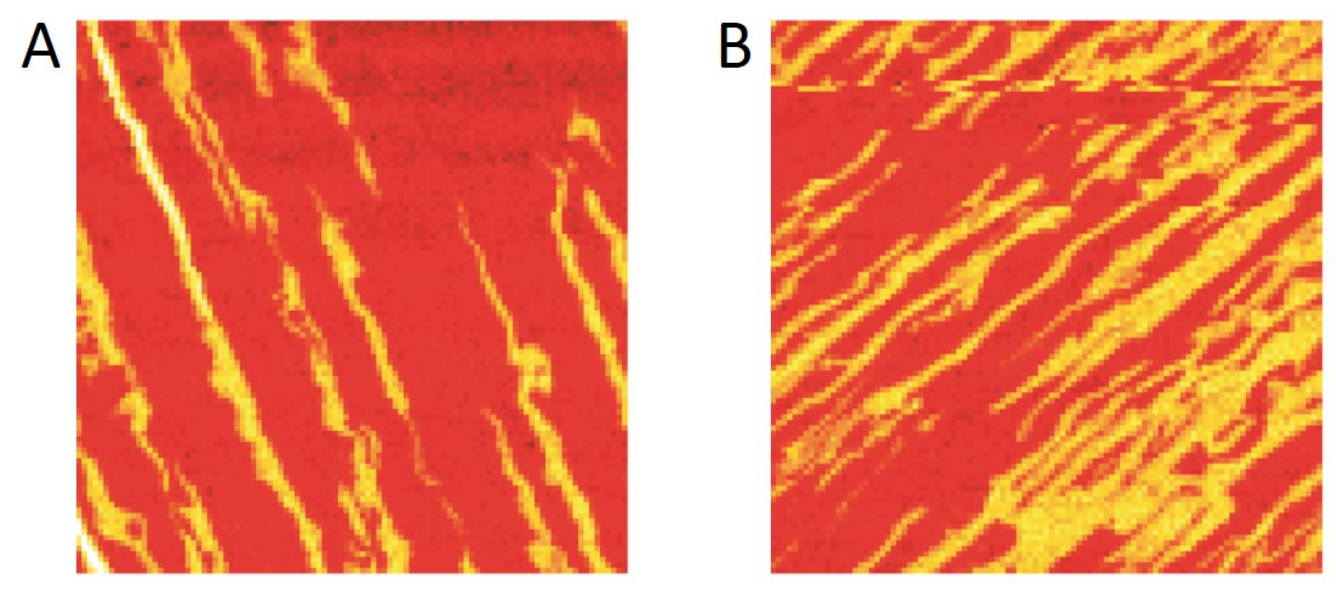

Figure S4. Reflectance maps on random areas $\left(30 \times 30 \mu \mathrm{m}^{2}\right)$ near the center of the pristine EG of $80 \%$ and $55 \%$ monolayer content ( $\mathbf{A}$ and $\mathbf{B}$ respectively). The monolayer and bilayer regions appear in red and yellow, respectively. 